\title{
Total transversals and total domination in uniform hypergraphs
}

\author{
Csilla Bujtás \\ Dept. of Computer Science and Systems Technology \\ University of Pannonia \\ H-8200 Veszprém, Egyetem u. 10, Hungary
}

bujtas@dcs.vein.hu

\author{
Michael A. Henning \\ Dept. of Mathematics \\ University of Johannesburg \\ Auckland Park, 2006 South Africa \\ mahenning@uj.ac.za
}

\author{
Zsolt Tuza \\ Dept. of Computer Science and Systems Technology \\ University of Pannonia \\ H-8200 Veszprém, Egyetem u. 10, Hungary \\ and \\ Alfréd Rényi Institute of Mathematics \\ Hungarian Academy of Sciences \\ H-1053 Budapest, Reáltanoda u. 13-15, Hungary \\ tuza@dcs.vein.hu \\ Anders Yeo \\ Engineering Systems and Design \\ Singapore University of Technology and Design \\ 20 Dover Drive Singapore, 138682, Singapore \\ and \\ Department of Mathematics \\ University of Johannesburg \\ Auckland Park, 2006 South Africa \\ andersyeo@gmail.com
}

Submitted: Nov 6, 2013; Accepted: Apr 4, 2014; Published: May 9, 2014

Mathematics Subject Classifications: 05C65

\begin{abstract}
In 2012, the first three authors established a relationship between the transversal number and the domination number of uniform hypergraphs. In this paper, we establish a relationship between the total transversal number and the total domination number of uniform hypergraphs. We prove tight asymptotic upper bounds on the total transversal number in terms of the number of vertices, the number of edges, and the edge size.
\end{abstract}

Keywords: Total domination; total transversal; hypergraph. 


\section{Introduction}

In this paper, we explore the study of total domination in hypergraphs. We establish a relationship between the transversal number and the total domination number of of uniform hypergraphs. We introduce the concept of a total transversal in a hypergraph and prove a general upper bound on the total domination number of a uniform hypergraph in terms of its total transversal number.

Hypergraphs are systems of sets which are conceived as natural extensions of graphs. A hypergraph $H=(V(H), E(H))$ is a finite set $V(H)$ of elements, called vertices, together with a finite multiset $E(H)$ of subsets of $V(H)$, called hyperedges or simply edges. If the hypergraph $H$ is clear from the context, we simply write $V=V(H)$ and $E=E(H)$. We shall use the notation $n_{H}=|V|$ (or $n(H)$ ) and $m_{H}=|E|$ (or $m(H)$ ), and sometimes simply $n$ and $m$ without subscript if the actual $H$ need not be emphasized, to denote the order and size of $H$, respectively. The edge set $E$ is often allowed to be a multiset in the literature, but in the present context we exclude multiple edges. Also, in the problems studied here, one may assume that $|V(e)| \geqslant 2$ holds for all $e \in E$. An isolated edge in $H$ is an edge in $H$ that does not intersect any other edge in $H$. A linear hypergraph is a hypergraph in which every two edges intersect in at most one vertex.

A $k$-edge in $H$ is an edge of size $k$. The hypergraph $H$ is said to be $k$-uniform if every edge of $H$ is a $k$-edge. The degree of a vertex $v$ in $H$, denoted by $d_{H}(v)$ or $d(v)$ if $H$ is clear from the context, is the number of edges of $H$ which contain $v$. A vertex of degree $k$ is called a degree- $k$ vertex. The number of degree- 1 vertices in $H$ is denoted by $n_{1}(H)$. The minimum degree among the vertices of $H$ is denoted by $\delta(H)$ and the maximum degree by $\Delta(H)$.

Two vertices $x$ and $y$ in $H$ are adjacent if there is an edge $e$ of $H$ such that $\{x, y\} \subseteq$ $V(e)$. The open neighborhood of a vertex $v$ in $H$, denoted $N_{H}(v)$ or simply $N(v)$ if $H$ is clear from the context, is the set of all vertices different from $v$ that are adjacent to $v$. We call a vertex in $N(v)$ a neighbor of $v$. Two vertices $x$ and $y$ in $H$ are connected if there is a sequence $x=v_{0}, v_{1}, v_{2} \ldots, v_{k}=y$ of vertices of $H$ in which $v_{i-1}$ is adjacent to $v_{i}$ for $i=1,2, \ldots, k$. A connected hypergraph is a hypergraph in which every pair of vertices are connected. A maximal connected subhypergraph of $H$ is a component of $H$. Thus, no edge in $H$ contains vertices from different components.

For a hypergraph $H$, the open neighborhood hypergraph of $H$, denoted by $\mathrm{ONH}(H)$, is the hypergraph with vertex set $V(H)$ and edge set $\left\{N_{H}(v) \mid v \in V(H)\right\}$ consisting of the open neighborhoods of vertices of $V(H)$ in $H$.

A subset $T$ of vertices in a hypergraph $H$ is a transversal (also called vertex cover or hitting set in many papers) if $T$ has a nonempty intersection with every edge of $H$. The transversal number $\tau(H)$ of $H$ is the minimum size of a transversal in $H$. A strong transversal, often called a 2-transversal, in $H$ is a transversal that contains at least two vertices from every edge in $H$. The strong transversal number $\tau_{s}(H)$ of $H$ is the minimum size of a strong transversal in $H$. Transversals in hypergraphs are well studied in the literature (see, for example, $[3,5,7,12,13,14,15,16,21,22,23]$ ).

We define a total transversal in $H$ to be transversal $T$ in $H$ with the additional property

THE ELECTRONiC JOURnal of COMBINATORICs 21(2) (2014), \#P2.24 
that every vertex in $T$ has at least one neighbor in $T$, and we define the total transversal number $\tau_{t}(H)$ of $H$ to be the minimum size of a total transversal in $H$.

For a subset $X \subset V(H)$ of vertices in $H$, we define $H-X$ to be the hypergraph obtained from $H$ by deleting the vertices in $X$ and all edges incident with $X$, and deleting resulting isolated vertices, if any. We note that if $T^{\prime}$ is a transversal in $H-X$, then $T^{\prime} \cup X$ is a transversal in $H$. If $X=\{x\}$, then we write $H-X$ simply as $H-x$.

A dominating set in a hypergraph $H=(V, E)$ is a subset of vertices $D \subseteq V$ such that for every vertex $v \in V \backslash D$ there exists an edge $e \in E$ for which $v \in e$ and $e \cap D \neq \emptyset$. Equivalently, every vertex $v \in V \backslash D$ is adjacent with a vertex in $D$. The domination number $\gamma(H)$ is the minimum cardinality of a dominating set in $H$. A vertex $v$ in $H$ is said to be a dominating vertex if it is adjacent to every other vertex in $H$. A total dominating set, abbreviated TD-set, in a hypergraph $H=(V, E)$ is a subset of vertices $D \subseteq V$ such that for every vertex $v \in V$ there exists an edge $e \in E$ for which $v \in e$ and $e \cap(D \backslash\{v\}) \neq \emptyset$. Equivalently, $D$ is a TD-set in $H$ if every vertex in $H$ is adjacent with a vertex in $D$. The total domination number $\gamma_{t}(H)$ is the minimum cardinality of a TD-set in $H$. A TD-set in $H$ of cardinality $\gamma_{t}(H)$ is called a $\gamma_{t}(H)$-set.

While domination and total domination in graphs is very well studied in the literature (see, for example, $[8,9,10,17]$ ), domination in hypergraphs was introduced relatively recently by Acharya [1] and studied further in $[2,4,11,18,19]$ and elsewhere.

An edge-cover in a graph $G$ is a set of edges such that every vertex in $G$ is incident with at least one edge in the edge-cover. We define a total edge-cover in $G$ to be an edgecover that induces a subgraph with no isolated edge. We let $\operatorname{ec}_{t}(G)$ denote the minimum cardinality of a total edge-cover in $G$. A 2-section graph, $(H)_{2}$, of a hypergraph $H$ is defined as the graph with the same vertex set as $H$ and in which two edges are adjacent in $(H)_{2}$ if and only if they belong to a common edge in $H$.

The interplay between total domination in graphs and transversals in hypergraphs has been studied in several papers (see, for example, [13, 14, 22]). The first three authors [4] establish a relationship between the transversal number and the domination number of uniform hypergraphs. In the present work, we establish a relationship between the total transversal number and the total domination number of uniform hypergraphs.

\subsection{Key Definitions}

We shall need the following definitions.

Definition 1. For an integer $k \geqslant 2$, let $\mathcal{H}_{k}$ be the class of all $k$-uniform hypergraphs containing no isolated vertices or isolated edges or multiple edges. Further, for $k \geqslant 3$ let $\mathcal{H}_{k}^{*}$ consist of all hypergraphs in $\mathcal{H}_{k}$ that have no two edges intersecting in $k-1$ vertices. We note that $\mathcal{H}_{k}^{*}$ is a proper subclass of $\mathcal{H}_{k}$.

Definition 2. For an integer $k \geqslant 2$, let

$$
b_{k}=\sup _{H \in \mathcal{H}_{k}} \frac{\tau_{t}(H)}{n_{H}+m_{H}} .
$$




\section{Main Results}

We shall prove the following upper bounds on the total domination number of a uniform hypergraph in terms of its total transversal number, order and size. A proof of Theorem 3 is presented in Section 5.1.

Theorem 3. For $k \geqslant 3$, if $H \in \mathcal{H}_{k}$, then $\gamma_{t}(H) \leqslant\left(\max \left\{\frac{2}{k+1}, b_{k-1}\right\}\right) n_{H}$.

In view of Theorem 3, it is of interest to determine the value of $b_{k}$ for $k \geqslant 2$. A proof of Theorem 4 is presented in Section 5.2.

Theorem 4. $b_{2}=\frac{2}{5}, b_{3}=\frac{1}{3}$, and $b_{4} \leqslant \frac{1}{3}$. Further for $k \geqslant 5$, we have $b_{k} \leqslant \frac{2}{7}$.

By Theorem 4, we observe that

$$
b_{k-1} \leqslant \frac{2}{k+1} \quad \text { for } k \in\{3,4,5,6\} .
$$

Hence as a consequence of Theorem 3 and Theorem 4, and the well-known fact (see, [6]) that if $H \in \mathcal{H}_{2}$, then $\gamma_{t}(H) \leqslant 2 n_{H} / 3$, we have the following result. The sharpness of the bound in Theorem 5 is shown in Observation 17 in Section 3.

Theorem 5. For $k \in\{2,3,4,5,6\}$, if $H \in \mathcal{H}_{k}$, then $\gamma_{t}(H) \leqslant 2 n_{H} /(k+1)$, and this bound is sharp.

The following result is a strengthening of the upper bound of Theorem 3 if we restrict the edges to intersect in at most $k-2$ vertices. A proof of Theorem 6 is presented in Section 5.3

Theorem 6. For $k \geqslant 4$, if $H \in \mathcal{H}_{k}^{*}$, then $\gamma_{t}(H) \leqslant\left(\max \left\{\frac{2}{k+2}, b_{k-1}\right\}\right) n_{H}$.

Corollary 7. For $k \geqslant 4$, if $H \in \mathcal{H}_{k}^{*}$, then $\gamma_{t}(H) \leqslant n_{H} / 3$.

The following result establishes a tight asymptotic bound on $b_{k}$ for $k$ sufficiently large. A proof of Theorem 8 is presented in Section 6.

Theorem 8. For all $k \geqslant 2, b_{k}=(1+\mathrm{o}(1)) \frac{\ln (k)}{k}$.

Theorem 8 implies that the inequality $b_{k-1} \leqslant 2 /(k+1)$ is not valid when $k$ is large enough. Using a result due to Alon [3], the first three authors proved the following result.

Theorem 9. ([4]) For all $k \geqslant 3$,

$$
\sup _{H \in \mathcal{H}_{k}} \frac{\gamma(H)}{n_{H}}=(1+\mathrm{o}(1)) \frac{\ln (k-1)}{k-1} .
$$


By definition, $\gamma_{t}(H) \geqslant \gamma(H)$ for every hypergraph $H$ with no isolated vertex. Hence as a consequence of Theorem 3, Theorem 8 and Theorem 9 , we have the following result.

Theorem 10. For all $k \geqslant 3$,

$$
\sup _{H \in \mathcal{H}_{k}} \frac{\gamma_{t}(H)}{n_{H}}=(1+\mathrm{o}(1)) \frac{\ln (k-1)}{k-1}=(1+\mathrm{o}(1)) \frac{\ln (k)}{k} .
$$

We remark that Theorem 10 implies that Theorem 5 is not true for large $k$.

\section{Known Results and Observations}

Cockayne et al. [6] established the following bound on the total domination number of a connected graph in terms of its order.

Theorem 11. ([6]) If $G$ is a connected graph of order $n \geqslant 3$, then $\gamma_{t}(G) \leqslant 2 n / 3$.

We shall need the following result due to Kelmans and Mubayi [20].

Theorem 12. ([20]) A cubic graph $G$ contains at least $\lceil|V(G)| / 4\rceil$ vertex disjoint $P_{3}$ 's.

The following result shows that the total domination number of a hypergraph $H$ is precisely the total domination of its 2-section graph and the transversal number of its open neighborhood hypergraph.

Observation 13. Let $H$ be a hypergraph with no isolated vertex. Then the following holds.

(a) $\gamma_{t}(H)=\gamma_{t}\left((\mathcal{H})_{2}\right)$.

(b) $\gamma_{t}(H)=\tau(\mathrm{ONH}(H))$.

Proof. (a) Part (a) follows readily from the fact that two vertices in $H$ are adjacent in $H$ if and only if they are adjacent in the 2-section graph $(H)_{2}$ of $H$.

(b) On the one hand, every TD-set in $H$ contains a vertex from the open neighborhood of each vertex in $H$, and is therefore a transversal in $\mathrm{ONH}$, implying that $\tau(\mathrm{ONH}(H)) \leqslant$ $\gamma_{t}(H)$. On the other hand, every transversal in ONH contains a vertex from the open neighborhood of each vertex of $H$, and is therefore a TD-set in $G$, implying that $\gamma_{t}(H) \leqslant$ $\tau(\mathrm{ONH}(H))$. Consequently, $\gamma_{t}(H)=\tau(\mathrm{ONH}(H))$.

We shall need the following properties of hypergraphs in the family $\mathcal{H}_{k}$.

Observation 14. For $k \geqslant 2$, if $H \in \mathcal{H}_{k}$, then the following hold.

(a) $n_{H} \geqslant k+1, m_{H} \geqslant 2$ and $\Delta(H) \geqslant 2$.

(b) $2 n_{H}-n_{1}(H) \geqslant 2 k$. 
Proof. Part (a) is immediate from the definition of the family $\mathcal{H}_{k}$. To prove Part (b), let $n_{\geqslant 2}(H)$ denote the number of vertices in $H$ of degree at least 2. Let $e$ and $f$ be any two intersecting edges in $H$ and suppose they intersect in $\ell$ vertices, and so $|e \cup f|=2 k-\ell$. Then, $n_{H} \geqslant 2 k-\ell \geqslant 2 k-n_{\geqslant 2}(H)$, or, equivalently, $2 n_{H}-n_{1}(H)=n_{H}+n_{\geqslant 2}(H) \geqslant 2 k$.

In order to state our next result, we first define two families of hypergraphs.

Definition 15. For $k \geqslant 2$, let $H$ be obtained from a hypergraph $F \in \mathcal{H}_{k}$ as follows. For each vertex $v$ in $F$, add $k$ new vertices $v_{1}, v_{2}, \ldots, v_{k}$ and two new $k$-edges $\left\{v, v_{1}, \ldots, v_{k-1}\right\}$ and $\left\{v_{1}, v_{2}, \ldots, v_{k}\right\}$. Let $\mathcal{F}_{k}$ denote the family of all such hypergraphs $H$.

Definition 16. For $k \geqslant 3$, let $H$ be obtained from a hypergraph $F \in \mathcal{H}_{k}^{*}$ as follows. For each vertex $v$ in $F$, add $k+1$ new vertices $v_{1}, v_{2}, \ldots, v_{k+1}$ and two new $k$-edges $\left\{v, v_{1}, v_{2}, \ldots, v_{k-1}\right\}$ and $\left\{v_{2}, v_{3}, \ldots, v_{k+1}\right\}$. Let $\mathcal{F}_{k}^{*}$ denote the family of all such hypergraphs $H$.

Observation 17. The following holds.

(a) For $k \geqslant 2$, if $H \in \mathcal{F}_{k}$, then $\gamma_{t}(H)=2 n_{H} /(k+1)$.

(b) For $k \geqslant 3$, if $H \in \mathcal{F}_{k}^{*}$, then $\gamma_{t}(H)=2 n_{H} /(k+2)$.

Proof. For $k \geqslant 2$, let $H \in \mathcal{F}_{k}$ be constructed as in Definition 15. Then, $H \in \mathcal{H}_{k}$ and $n_{H}=$ $(k+1) n_{F}$. Every TD-set in $H$ contains at least two vertices in $\left\{v, v_{1}, v_{2}, \ldots, v_{k}\right\}$, implying that $\gamma_{t}(H) \geqslant 2 n_{F}$. However, the set $V(F) \cup T$, where $|T|=n_{F}$ and $T \subseteq V(H) \backslash V(F)$ consists of one added neighbor of each vertex in $V(F)$, is a TD-set in $H$, implying that $\gamma_{t}(H) \leqslant 2 n_{F}$. Consequently, $\gamma_{t}(H)=2 n_{F}=2 n_{H} /(k+1)$. For $k \geqslant 3$, let $H \in \mathcal{F}_{k}^{*}$ be constructed as in Definition 16. Then, $H \in \mathcal{H}_{k}^{*}$ and $\gamma_{t}(H)=2 n_{F}=2 n_{H} /(k+2)$.

\section{Preliminary Result}

We show that total transversals of a 2-regular hypergraph $H$ correspond to total edgecovers in the dual multigraph, $G_{H}$, of $H$, where the vertices of $G_{H}$ are the edges of $H$ and the edges of $G_{H}$ correspond to the vertices of $H$ : if a vertex of $H$ is contained in the edges $e$ and $f$ of $H$, then the corresponding edge of the multigraph $G_{H}$ joins vertices $e$ and $f$ of $G_{H}$.

Lemma 18. If $H$ is a linear 2-regular hypergraph and $G_{H}$ is the dual of $H$, then $\tau_{t}(H)=$ $\mathrm{ec}_{t}\left(G_{H}\right)$.

Proof. By the linearity of $H$, the multigraph $G_{H}$ is in fact a graph. Let $T$ be a total transversal in $H$. Then the edges of $G_{H}$ corresponding to vertices in $T$ form an edgecover in $G_{H}$. Further if $u$ and $v$ are neighbors in $H$ that belong to $T$, then the edges in $G_{H}$ corresponding to the vertices $u$ and $v$ in $H$ have a vertex in common, namely the edge in $H$ that contains both $u$ and $v$. This implies that the edge-cover in $G_{H}$ corresponding to the total transversal $T$ in $H$ is a total edge-cover in $G_{H}$. Similarly, every total edge-cover in $G_{H}$ corresponds to a total transversal in $H$. Therefore, $\tau_{t}(H)=\mathrm{ec}_{t}\left(G_{H}\right)$. 


\section{Proofs of Main Results}

\subsection{Proof of Theorem 3}

In this section, we present a proof of Theorem 3. Recall its statement.

Theorem 3. For $k \geqslant 3$, if $H \in \mathcal{H}_{k}$, then $\gamma_{t}(H) \leqslant\left(\max \left\{\frac{2}{k+1}, b_{k-1}\right\}\right) n_{H}$.

Proof of Theorem 3. Suppose to the contrary that the theorem is not true. Let $H \in \mathcal{H}_{k}$ be a counterexample with $n_{H}+m_{H}$ a minimum. In what follows we present a series of claims describing some structural properties of $H$ which culminate in the implication of its non-existence.

Claim 19. The following properties hold in the hypergraph $H$.

(a) $H$ is connected.

(b) The deletion of any edge in $H$ creates an isolated vertex or an isolated edge.

(c) There is no dominating vertex in $H$.

Proof of Claim 19. Part (a) is immediate from the minimality of $H$. Part (b) is also immediate since the deletion of an edge cannot decrease the total domination number. To prove Part (c), suppose that $H$ contains a dominating vertex $v$. The vertex $v$ and any one of its neighbors forms a TD-set in $H$, implying that $\gamma_{t}(H)=2$. As $H \in \mathcal{H}_{k}$, there is no isolated vertex or isolated edge in $H$, implying that $n_{H} \geqslant k+1$. Hence, $\gamma_{t}(H) \leqslant 2 n_{H} /(k+1)$, contradicting the fact that $H$ is a counterexample to the theorem. This proves Part (c). (口)

Claim 20. Every edge in $H$ contains at least one degree-1 vertex.

Proof of Claim 20. Suppose to the contrary that there is an edge $e$ that does not contain any degree- 1 vertices. Thus every vertex contained in $e$ has degree at least 2 in $H$. By Claim 19(b), there is therefore an edge, $e_{1}$, which would become isolated after the deletion of the edge $e$ from $H$. Thus, every vertex in $e \cap e_{1}$ has degree 2 in $H$, while every vertex in $e_{1} \backslash e$ has degree 1 in $H$. Let $v \in e \cap e_{1}$. Then, $d_{H}(v)=2$. By Claim 19(a), $H$ is connected and by Claim 19(c), the vertex $v$ is not a dominating vertex of $H$, implying that there exists an edge, $e_{2}$, such that $v \notin e_{2}$ but $e_{2}$ intersects $e$. Since $e \neq e_{2}$ and $v \notin e_{2}$, we note that $e_{1} \cap e_{2}=\emptyset$. Let $u \in e \cap e_{2}$ and note that $u \notin e_{1}$.

Initially we set $T=\emptyset$ and we now construct a hypergraph $H^{\prime}$ from $H$ as follows. We delete all edges incident with $u$ or $v$ or with both $u$ and $v$ and we delete any resulting isolated vertices. Further we add both vertices $u$ and $v$ to the set $T$. We note that the edges $e$ and $e_{1}$ are both deleted, implying that every vertex in $e_{1}$ becomes an isolated vertex. Further since we remove all edges incident with $u$, the vertex $u$ becomes an isolated vertex. We therefore delete at least $k+1$ vertices and we add two vertices to $T$. If this process creates an isolated edge, then such an isolated edge necessarily contains a vertex that is adjacent to at least one of $u$ and $v$ (for otherwise it would be an isolated edge in $H$, a contradiction). From each such isolated edge $f$, if any, we choose one vertex that is a neighbor of $u$ or $v$ and add it into $T$, and delete the $k$ vertices in $f$. Hence, 
$|T|=2+\ell$, where $\ell \geqslant 0$ denotes the number of isolated edges created when removing $u$ and $v$.

Let $n^{\prime}$ denote the number of vertices in $H$ that are not deleted in the process (possibly, $\left.n^{\prime}=0\right)$. At least $k+1+k \ell$ vertices were deleted from $H$. Thus, $n^{\prime} \leqslant n_{H}-k-1-k \ell$, implying that

$$
\begin{aligned}
\left(\frac{2}{k+1}\right)\left(n_{H}-n^{\prime}\right) & \geqslant\left(\frac{2}{k+1}\right)(k+1+k \ell) \\
& =2+\left(\frac{2 k}{k+1}\right) \ell \\
& \geqslant 2+\ell \\
& =|T| .
\end{aligned}
$$

If $n^{\prime}=0$, then the set $T$ is a TD-set in $H$, implying that $\gamma_{t}(H) \leqslant|T| \leqslant 2 n_{H} /(k+1)$, a contradiction. Hence, $n^{\prime}>0$. Let $H^{\prime}$ denote the resulting hypergraph on these $n^{\prime}$ vertices. Let $H^{\prime}$ have size $m^{\prime}$. By construction, the hypergraph $H^{\prime}$ is in the family $\mathcal{H}_{k}$. In particular, we note that $n^{\prime} \geqslant k+1$. By the minimality of $H$, we have that

$$
\gamma_{t}\left(H^{\prime}\right) \leqslant\left(\max \left\{\frac{2}{k+1}, b_{k-1}\right\}\right) n^{\prime} .
$$

Let $T^{\prime}$ be a $\gamma_{t}\left(H^{\prime}\right)$-set and note that the set $T \cup T^{\prime}$ is a TD-set of $H$. Suppose that $2 /(k+1) \geqslant b_{k-1}$. Then, $\left|T^{\prime}\right| \leqslant 2 n^{\prime} /(k+1)$, and so

$$
\gamma_{t}(H) \leqslant\left|T \cup T^{\prime}\right| \leqslant\left(\frac{2}{k+1}\right)\left(n_{H}-n^{\prime}\right)+\left(\frac{2}{k+1}\right) n^{\prime}=\left(\frac{2}{k+1}\right) n_{H},
$$

a contradiction. Hence, $2 /(k+1)<b_{k-1}$. Thus, $\left|T^{\prime}\right| \leqslant b_{k-1} n^{\prime}$, and so

$$
\gamma_{t}(H) \leqslant\left|T \cup T^{\prime}\right| \leqslant\left(\frac{2}{k+1}\right)\left(n_{H}-n^{\prime}\right)+b_{k-1} n^{\prime}<b_{k-1}\left(n_{H}-n^{\prime}\right)+b_{k-1} n^{\prime}=b_{k-1} n_{H},
$$

a contradiction. This completes the proof of Claim 20. (ㅁ)

We now return to the proof of Theorem 3. By Claim 20, every edge in $H$ contains at least one degree- 1 vertex. If there are two edges, $f_{1}$ and $f_{2}$, in $H$ that intersect in $k-1$ vertices, then for $j \in\{1,2\}$, the edge $f_{j}$ contains exactly one vertex, $v_{j}$ say, not in $f_{3-j}$ and this vertex has degree 1 in $H$. Thus if we delete the vertices $v_{1}$ and $v_{2}$ from $H$, then we would create a multiple edge, namely $f_{1}^{\prime}=f_{1} \backslash\left\{v_{1}\right\}$ and $f_{2}^{\prime}=f_{2} \backslash\left\{v_{2}\right\}$. Let $H^{\prime}$ be the hypergraph obtained from $H$ by deleting exactly one degree- 1 vertex from each edge and by replacing resulting multiple edges, if any, by single edges. Let $H^{\prime}$ have order $n^{\prime}$ and size $m^{\prime}$. Then, $n^{\prime}=n_{H}-m_{H}$ and $m^{\prime} \leqslant m_{H}$. Thus, $n^{\prime}+m^{\prime} \leqslant n_{H}$.

Claim 21. $H^{\prime} \in \mathcal{H}_{k-1}$ and $\tau_{t}\left(H^{\prime}\right) \leqslant b_{k-1} n_{H}$. 
Proof of Claim 21. If $H^{\prime}$ contains an isolated edge, then every vertex in such an isolated edge would be a dominating vertex in $H$, contradicting Claim 19(c). Hence, $H^{\prime}$ contains no isolated edge. By construction, $H^{\prime}$ has no multiple edges and no isolated vertices. Therefore, $H^{\prime} \in \mathcal{H}_{k-1}$. We note that $k-1 \geqslant 2$. By Definition 2 we have that $\tau_{t}\left(H^{\prime}\right) \leqslant\left(n^{\prime}+m^{\prime}\right) b_{k-1} \leqslant b_{k-1} n_{H} \cdot(\square)$

Claim 22. $\tau_{t}\left(H^{\prime}\right)=\gamma_{t}(H)$.

Proof of Claim 22. Among all $\gamma_{t}(H)$-sets, let $S$ be chosen to contain as few vertices of degree 1 in $H$ as possible. Suppose that $S$ contains a degree-1 vertex, $x$, in $H$. Let $e_{x}$ be the edge containing $x$. By the minimality of the set $S$, the set $S_{x}=S \backslash\{x\}$ is not a TD-set in $H$. Let $y$ be a vertex in $S$ that is adjacent to $x$ in $H$. Then, $y \in e_{x}$. If $y$ is adjacent to a vertex of $S_{x}$, then the set $S_{x}$ would be a TD-set in $H$, a contradiction. Hence, $y$ is adjacent to no vertex of $S$ except for the vertex $x$. Since $H$ contains no dominating vertex and since $H$ has no isolated edge, there exists a neighbor, $w$ say, of $y$ that has degree at least 2 in $H$. But then $S_{x} \cup\{w\}$ is a TD-set of $H$ of cardinality $|S|=\gamma_{t}(H)$ that contains fewer degree-1 vertices than does $S$, contradicting our choice of the set $S$. Therefore, $S$ contains no vertices of degree 1, implying that $S \subseteq V\left(H^{\prime}\right)$. Further if $S$ is not a transversal in $H$, then let $e^{\prime}$ be an edge in $H$ not intersected by $S$. But since $e^{\prime}$ contains a degree-1 vertex, such a vertex would not be (totally) dominated by $S$ in $H$, a contradiction. Hence, $S$ is a transversal in $H$. Further since every vertex in the TD-set $S$ has a neighbor in $H$ that belongs to $S$, the set $S$ is in fact a total transversal of $H$. Since $S \subseteq V\left(H^{\prime}\right)$, the set $S$ is therefore also a total transversal of $H^{\prime}$, implying that $\tau_{t}\left(H^{\prime}\right) \leqslant \gamma_{t}(H)$. Conversely, every total transversal in $H^{\prime}$ is a TD-set in $H^{\prime}$ and therefore also in $H$, implying that $\gamma_{t}(H) \leqslant \tau_{t}\left(H^{\prime}\right)$. Consequently, $\tau_{t}\left(H^{\prime}\right)=\gamma_{t}(H)$.

By Claim 21 and Claim 22, we have that $\gamma_{t}(H) \leqslant b_{k-1} n_{H}$, a contradiction. This completes the proof of Theorem 3 .

\subsection{Proof of Theorem 4}

In this section, we present a proof of Theorem 4. We first consider the family $\mathcal{H}_{2}$.

Theorem 23. If $H \in \mathcal{H}_{2}$, then $\tau_{t}(H) \leqslant 2\left(n_{H}+m_{H}\right) / 5$.

Proof of Theorem 23. Suppose to the contrary that the theorem is not true. Let $H \in \mathcal{H}_{2}$ be a counterexample with $n_{H}+m_{H}$ a minimum. Clearly, $H$ is connected. By Observation 14, we have that $n_{H} \geqslant 3, m_{H} \geqslant 2$ and $\Delta(H) \geqslant 2$. If $\tau_{t}(H)=2$, then the result is immediate. Hence we may assume that $\tau_{t}(H) \geqslant 3$. Let $x$ be a vertex of maximum degree in $H$. Since $\tau_{t}(H) \geqslant 3$, there is a neighbor $y$ of $x$ that is not isolated in $H-x$. We delete the vertices $x$ and $y$ and all edges incident with $x$ or $y$, together with any resulting isolated vertices, if any, and let $T=\{x, y\}$. Further if this process creates an isolated edge, $e$, then such an isolated edge necessarily contains a vertex that is adjacent to $x$ or $y$, for otherwise the edge $e$ would be an isolated edge in $H$, a contradiction. From each such isolated edge $e$, if any, we choose one vertex that is a neighbor of $x$ or $y$ and add it to the 
set $T$, and delete the two vertices in $e$. Suppose that $\ell \geqslant 0$ isolated edges were created when $x$ and $y$ are deleted. Then, $|T|=2+\ell$ and at least $2+2 \ell$ vertices and at least $3+\ell$ edges were deleted. Let $H^{\prime}$ denote the resulting graph. Thus, if $H^{\prime}$ has $n^{\prime}$ vertices and $m^{\prime}$ edges, then $n^{\prime}+m^{\prime} \leqslant n_{H}+m_{H}-(5+3 \ell)$. Since $H$ is a minimum counterexample, we have that $\tau_{t}\left(H^{\prime}\right) \leqslant 2\left(n^{\prime}+m^{\prime}\right) / 5$, implying that

$$
\begin{aligned}
\tau_{t}(H) & \leqslant \tau_{t}\left(H^{\prime}\right)+|T| \\
& \leqslant \frac{2}{5}\left(n_{H}+m_{H}-5-3 \ell\right)+2+\ell \\
& \leqslant \frac{2}{5}\left(n_{H}+m_{H}\right)-\frac{\ell}{5} \\
& \leqslant \frac{2}{5}\left(n_{H}+m_{H}\right),
\end{aligned}
$$

contradicting the fact that $H$ is a counterexample.

As an immediate consequence of Theorem 23, we have that $b_{2} \leqslant 2 / 5$. Taking $H$ to be a path $P_{3}$ on three vertices, we note that $H \in \mathcal{H}_{2}$ and $\tau_{t}(H)=2=2\left(n_{H}+m_{H}\right) / 5$, implying that $b_{2} \geqslant 2 / 5$. Consequently, $b_{2}=2 / 5$. This can also be seen by considering the cycle of order five, $C_{5}$, instead of $P_{3}$, as $\tau_{t}\left(C_{5}\right)=4$. We state this formally as follows.

Corollary 24. $b_{2}=2 / 5$.

We next consider the family $\mathcal{H}_{k}$, where $k \geqslant 3$.

Theorem 25. For $k \geqslant 3$, if $H \in \mathcal{H}_{k}$, then $\tau_{t}(H) \leqslant\left(n_{H}+m_{H}\right) / 3$.

Proof of Theorem 25. Suppose to the contrary that the theorem is not true. Let $H \in \mathcal{H}_{k}$ be a counterexample with $n_{H}+m_{H}$ a minimum. Clearly, $H$ is connected since otherwise the theorem holds for each component of $H$ and therefore also for $H$, a contradiction. By Observation 14, we have that $n_{H} \geqslant k+1, m_{H} \geqslant 2$ and $\Delta(H) \geqslant 2$. In what follows we present a series of claims describing some structural properties of $H$ which culminate in the implication of its non-existence.

Claim A. $\tau_{t}(H) \geqslant 3$ and no vertex is incident with every edge in $H$.

Proof of Claim A. Suppose to the contrary that $\tau_{t}(H)<3$. Then, $\tau_{t}(H)=2$. Since $n_{H}+m_{H} \geqslant k+3 \geqslant 6$, we therefore have that $\tau_{t}(H)=2 \leqslant\left(n_{H}+m_{H}\right) / 3$, contradicting the fact that $H$ is a counterexample. Hence, $\tau_{t}(H) \geqslant 3$.

If there is a vertex $v$ incident with every edge in $H$, then the vertex $v$ and one of its neighbors form a total transversal in $H$, implying that $\tau_{t}(H)=2$, a contradiction. Hence, no vertex is incident with every edge in $H$. (口)

Claim B. If $X$ is a set of vertices in $H$, such that every vertex in $X$ is adjacent to some other vertex of $X$, then $n(H-X)+m(H-X)>n_{H}+m_{H}-3|X|$.

Proof of Claim B. Suppose to the contrary that there exists a subset $X \subset V(H)$ such that every vertex in $X$ is adjacent to some other vertex of $X$ but $n(H-X)+m(H-X) \leqslant$ $n_{H}+m_{H}-3|X|$. Let $H^{\prime}=H-X$. By supposition, $n\left(H^{\prime}\right)+m\left(H^{\prime}\right) \leqslant n_{H}+m_{H}-3|X|$. 
Let $e_{1}, \ldots, e_{\ell}$, where $\ell \geqslant 0$, be the isolated edges in $H^{\prime}$. Since $H$ contains no isolated edge, each isolated edge in $H^{\prime}$ contains a vertex of degree at least 2 in $H$. For each $i=1, \ldots, \ell$, let $z_{i} \in e_{i}$ be chosen so that $d_{H}\left(z_{i}\right) \geqslant 2$, and let $X^{*}=X \cup\left\{z_{1}, \ldots, z_{\ell}\right\}$. We note that every vertex in $X^{*}$ is adjacent to a vertex in $X \subseteq X^{*}$.

Let $H^{*}=H-X^{*}$. By construction, $H^{*} \in \mathcal{H}_{k}$. Moreover, $n\left(H^{*}\right)=n\left(H^{\prime}\right)-k \ell$ and $m\left(H^{*}\right)=m\left(H^{\prime}\right)-\ell$. By the minimality of $H$, we have that $\tau_{t}\left(H^{*}\right) \leqslant\left(n\left(H^{*}\right)+m\left(H^{*}\right)\right) / 3$. Since every $\tau_{t}\left(H^{*}\right)$-set can be extended to a total transversal of $H$ by adding to it the set $X^{*}$, and since $k \geqslant 3$, we have that

$$
\begin{aligned}
\tau_{t}(H) & \leqslant \tau_{t}\left(H^{*}\right)+\left|X^{*}\right| \\
& \leqslant \frac{1}{3}\left(n\left(H^{*}\right)+m\left(H^{*}\right)\right)+|X|+\ell \\
& =\frac{1}{3}\left(n\left(H^{\prime}\right)-k \ell+m\left(H^{\prime}\right)-\ell\right)+|X|+\ell \\
& \leqslant \frac{1}{3}\left(n_{H}+m_{H}-3|X|-k \ell-\ell\right)+|X|+\ell \\
& \leqslant \frac{1}{3}\left(n_{H}+m_{H}\right),
\end{aligned}
$$

contradicting the fact that $H$ is a counterexample. (口)

Claim C. $\Delta(H)=2$.

Proof of Claim C. Suppose to the contrary that $\Delta(H) \geqslant 3$. Let $x$ be a vertex of maximum degree in $H$. By Claim A, the vertex $x$ is not incident with every edge in $H$. Hence since $H$ is connected, there exists an edge, $e$, that contains a neighbor, $y$, of $x$ but does not contain $x$. Let $X=\{x, y\}$ and note that $n(H-X) \leqslant n_{H}-2$ and $m(H-X) \leqslant m_{H}-4$. As $x$ and $y$ are adjacent in $H$, we obtain a contradiction to Claim B. (ㅁ)

Claim D. $H$ is 2-regular.

Proof of Claim D. Suppose that there exists a vertex $v_{1}$ of degree 1 in $H$. Let $e_{1}$ be the edge incident with $v_{1}$. Since $H$ has no isolated edge, let $e_{2}$ be an edge intersecting $e_{1}$, and let $v_{2} \in e_{1} \cap e_{2}$. By Claim A, the vertex $v_{2}$ is not incident with every edge in $H$. Hence there exists an edge, $e_{3}$, not containing $v_{2}$ that intersects $e_{1}$ or $e_{2}$ in a vertex $v_{3}$. Let $X=\left\{v_{2}, v_{3}\right\}$ and note that the vertices $v_{1}, v_{2}, v_{3}$ and the edges $e_{1}, e_{2}, e_{3}$ are removed from $H$ in order to create $H-X$. Therefore, $n(H-X) \leqslant n_{H}-3$ and $m(H-X) \leqslant m_{H}-3$, which as $v_{2}$ and $v_{3}$ are adjacent in $H$, contradicts Claim B. (口)

Claim E. $H$ is a linear hypergraph.

Proof of Claim E. By Claim D, $H$ is a 2-regular $k$-uniform hypergraph. Suppose that there are two edges $e$ and $f$ having two or more vertices in common. Let $v$ be a vertex in 
$e$ that does not belong to $e \cap f$. Since $H$ is 2-regular, there is an edge $g$ which contains $v$ but is different from $e$ or $f$. Let $u$ be a vertex in $e \cap f$. Since $u$ and $v$ belong to the common edge $e$, they are neighbors in $H$. Let $X=\{u, v\}$ and note that the vertices in $\{v\} \cup(e \cap f)$ and the edges $e, f, g$ are removed from $H$ in order to create $H-X$. Therefore, $n(H-X) \leqslant n_{H}-3$ and $m(H-X) \leqslant m_{H}-3$, which contradicts Claim B. (口)

By Claim D and Claim E, $H$ is a 2-regular $k$-uniform linear connected hypergraph.

Claim F. $k=3$

Proof of Claim F. Suppose to the contrary that $k \geqslant 4$. Then, $n_{H}=k m_{H} / 2 \geqslant 2 m_{H}$. We now consider the dual, $G_{H}$, of the hypergraph $H$. By the 2-regularity and the linearity of $H$, the dual $G_{H}$ is a graph. Since $H$ is $k$-uniform, the graph $G_{H}$ is $k$-regular. Further since $H$ is connected, so too is $G_{H}$. By construction, $G_{H}$ has order $n\left(G_{H}\right)=m_{H}$ and size $m\left(G_{H}\right)=n_{H}$. Let $T$ be a spanning tree in $G_{H}$. Since the set $E(T)$ of edges of $T$ forms a total edge-cover in $G_{H}$ and since $n_{H} \geqslant 2 m_{H}$, we have by Lemma 18 that $\tau_{t}(H)=\mathrm{ec}_{t}\left(G_{H}\right) \leqslant|E(T)|=n\left(G_{H}\right)-1=m_{H}-1<\frac{1}{3}\left(n_{H}+m_{H}\right)$, a contradiction.

By Claim D, E and $\mathrm{F}$, we have that $H$ is a 2-regular 3-uniform linear connected hypergraph. We now consider the dual, $G_{H}$, of the hypergraph $H$. We note that the dual, $G_{H}$, is a connected, cubic graph. Applying Theorem 12 to the cubic graph $G_{H}$, there exist at least $\left\lceil n\left(G_{H}\right) / 4\right\rceil$ vertex disjoint $P_{3}$ 's in $G_{H}$. Let $G_{1}, G_{2}, \ldots, G_{\ell}$ denote vertex disjoint subgraphs in $G_{H}$ each of which is isomorphic to $P_{3}$, such that $\ell \geqslant\left\lceil n\left(G_{H}\right) / 4\right\rceil \geqslant$ $m_{H} / 4$. If some vertex does not belong to one of these subgraphs $G_{1}, G_{2}, \ldots, G_{\ell}$, then the connectivity of $G_{H}$ implies that there is an edge, $e$, joining a vertex in $V\left(G_{i}\right)$ for some $i$, $1 \leqslant i \leqslant \ell$, and a vertex, $x$, not belonging to any subgraph $G_{1}, G_{2}, \ldots, G_{\ell}$. We now add the vertex $x$ and edge $e$ to the subgraph $G_{i}$. We continue this process until all vertices in $G_{H}$ belong to exactly one of the resulting subgraphs $G_{1}, G_{2}, \ldots, G_{\ell}$. The subgraph of $G_{H}$ induced by the edges in these $\ell$ subgraphs is a spanning forest, $F$, of $G_{H}$, that contains $\ell \geqslant m_{H} / 4$ components each of which contains at least three vertices.

Since every component of $F$ has order at least 3, the set $E(F)$ of edges of $F$ forms a total edge-cover in $G_{H}$. Since $n\left(G_{H}\right)=m_{H}$ and $\ell \geqslant m_{H} / 4$, we have that $|E(F)|=$ $n\left(G_{H}\right)-\ell \leqslant 3 m_{H} / 4$. Therefore, recalling that $n_{H}=3 m_{H} / 2$, we have by Lemma 18 that

$$
\tau_{t}(H)=\mathrm{ec}_{t}\left(G_{H}\right) \leqslant|E(F)| \leqslant \frac{3}{4} m_{H} \leqslant \frac{1}{3}\left(n_{H}+m_{H}\right),
$$

a contradiction. This completes the proof of Theorem 25 .

As an immediate consequence of Theorem 25 , we have that $b_{k} \leqslant 1 / 3$ for all $k \geqslant 3$. Taking $H$ to be the hypergraph of order $n_{H}=4$ and size $m_{H}=2$ where the two edges of $H$ intersect in two vertices, we note that $H \in \mathcal{H}_{3}$ and $\tau_{t}(H)=2=\left(n_{H}+m_{H}\right) / 3$, implying that $b_{3} \geqslant 1 / 3$. Consequently, $b_{3}=1 / 3$. As observed earlier, $b_{4} \leqslant 1 / 3$. We state this formally as follows.

Corollary 26. $b_{3}=\frac{1}{3}$ and $b_{4} \leqslant \frac{1}{3}$. 
We remark that the result of Theorem 25 can be strengthened slightly when $k \geqslant 4$, as the following result shows. We omit the proof (which is similar, but simpler, to the proof of Theorem 28 presented below).

Theorem 27. For $k \geqslant 4$, if $H \in \mathcal{H}_{k}$, then $6 \tau_{t}(H) \leqslant 2 n_{H}+2 m_{H}-n_{1}(H)$.

We next consider the family $\mathcal{H}_{k}$, where $k \geqslant 5$.

Theorem 28. For $k \geqslant 5$, if $H \in \mathcal{H}_{k}$, then $7 \tau_{t}(H) \leqslant 2 n_{H}+2 m_{H}-n_{1}(H)$.

Proof of Theorem 28. For $k \geqslant 5$ and all hypergraphs $H \in \mathcal{H}_{k}$, let

$$
\Theta(H)=2 n_{H}+2 m_{H}-n_{1}(H) .
$$

We wish to show that $7 \tau_{t}(H) \leqslant \Theta(H)$. Suppose to the contrary that the theorem is not true. Let $H \in \mathcal{H}_{k}$ be a counterexample with minimum $\Theta(H)$. Clearly, $H$ is connected since otherwise the theorem holds for each component of $H$ and therefore also for $H$, a contradiction. By Observation 14(a), we have that $n_{H} \geqslant k+1, m_{H} \geqslant 2$ and $\Delta(H) \geqslant 2$. By Observation 14(b), we have that $2 n_{H}-n_{1}(H) \geqslant 2 k$. In what follows we present a series of claims describing some structural properties of $H$ which culminate in the implication of its non-existence.

Claim I. $\tau_{t}(H) \geqslant 3$.

Proof of Claim I. Suppose that $\tau_{t}(H)<3$. Then, $\tau_{t}(H)=2$. Since $2 n_{H}-n_{1}(H) \geqslant 2 k$ and $m_{H} \geqslant 2$, we therefore have that $7 \tau_{t}(H)=14 \leqslant 2 k+4 \leqslant \Theta(H)$, contradicting the fact that $H$ is a counterexample. (口)

Claim II. If $X$ is a set of vertices in $H$, such that every vertex in $X$ is adjacent to some other vertex of $X$, then $\Theta(H-X)>\Theta(H)-7|X|$.

Proof of Claim II. Suppose to the contrary that exists a subset $X \subset V(H)$ such that every vertex in $X$ is adjacent to some other vertex of $X$ but $\Theta(H-X) \leqslant \Theta(H)-7|X|$. Let $H^{\prime}=H-X$. Let $e_{1}, \ldots, e_{\ell}$, where $\ell \geqslant 0$, be the isolated edges in $H^{\prime}$. Since $H$ contains no isolated edge, every isolated edge in $H^{\prime}$ contains a vertex of degree at least 2 in $H$ that is adjacent to a vertex of $X$ in $H$. For each $i=1, \ldots, \ell$, let $z_{i} \in e_{i}$ be chosen so that $d_{H}\left(z_{i}\right) \geqslant 2$, and let $X^{*}=\left\{z_{1}, \ldots, z_{\ell}\right\}$. We note that every vertex in $X \cup X^{*}$ is adjacent to some other vertex of $X$. We now consider the hypergraph $H^{*}=H^{\prime}-X^{*}$.

We note that $H^{*} \in \mathcal{H}_{k}$. When constructing $H^{*}$ from $H^{\prime}$ we deleted all $k \ell$ vertices from the $\ell$ isolated edges in $H^{\prime}$ and we deleted all $\ell$ isolated edges. Since each such deleted vertex has degree 1 in $H^{\prime}$, the contribution of the $k \ell$ deleted vertices from $H^{\prime}$ to the sum $2 n\left(H^{\prime}\right)-n_{1}\left(H^{\prime}\right)$ is $k \ell$. The contribution of the $\ell$ deleted edges to the sum $2 m\left(H^{\prime}\right)$ is $2 \ell$. By supposition, $\Theta\left(H^{\prime}\right) \leqslant \Theta(H)-7|X|$. Since $k \geqslant 5$, we therefore have that

$$
\begin{aligned}
\Theta\left(H^{*}\right) & =\Theta\left(H^{\prime}\right)-\ell(k+2) \\
& \leqslant \Theta\left(H^{\prime}\right)-7 \ell \\
& \leqslant(\Theta(H)-7|X|)-7 \ell \\
& =\Theta(H)-7|X|-7\left|X^{*}\right| .
\end{aligned}
$$


By the minimality of $\Theta(H)$, we have that $7 \tau_{t}\left(H^{*}\right) \leqslant \Theta\left(H^{*}\right)$. Every (minimum) total transversal in $H^{*}$ can be extended to a total transversal in $H$ by adding to the set $X \cup X^{*}$, implying that $\tau_{t}(H) \leqslant \tau_{t}\left(H^{*}\right)+|X|+\left|X^{*}\right|$. Hence,

$$
\begin{aligned}
7 \tau_{t}(H) & \leqslant 7 \tau_{t}\left(H^{*}\right)+7|X|+7\left|X^{*}\right| \\
& \leqslant \Theta\left(H^{*}\right)+7|X|+7\left|X^{*}\right| \\
& \leqslant \Theta(H)
\end{aligned}
$$

a contradiction. (口)

Claim III. $\Delta(H) \leqslant 3$.

Proof of Claim III. Suppose to the contrary that $\Delta(H) \geqslant 4$. Let $x$ be a vertex of maximum degree in $H$. Since $\tau_{t}(H) \geqslant 3$ by Claim I, and since $H$ is connected, there exists an edge, $e$, that contains a neighbor, $y$, of $x$ but does not contain $x$. Let $X=\{x, y\}$ and consider the hypergraph $H-X$. Since $d_{H}(x) \geqslant 4$ and $d_{H}(y) \geqslant 2$, the vertices $x$ and $y$ both contribute 2 to the sum $2 n(H)-n_{1}(H)$. Further since at least five distinct edges are deleted from $H$ when constructing $H-X$, the contribution of the deleted edges to the sum $2 m(H)$ is at least 10 . Hence, $\Theta(H-X) \leqslant \Theta(H)-14=\Theta(H)-7|X|$, contradicting Claim II.

Claim IV. $\Delta(H)=2$.

Proof of Claim IV. As observed earlier, $\Delta(H) \geqslant 2$. By Claim III, $\Delta(H) \leqslant 3$. Suppose to the contrary that $\Delta(H)=3$. Let $x$ be a vertex with $d_{H}(x)=3$ and consider the hypergraph $H^{\prime}=H-x$. Suppose that $d_{H^{\prime}}(y) \geqslant 2$ for some $y \in N_{H}(x)$. Let $X=\{x, y\}$ and consider the hypergraph $H-X$. Since $d_{H}(x)=3$ and $d_{H}(y)=3$, the vertices $x$ and $y$ both contribute 2 to the sum $2 n(H)-n_{1}(H)$. Further since five distinct edges are deleted from $H$ when constructing $H-X$, the contribution of the deleted edges to the sum $2 m(H)$ is 10 . Hence, $\Theta(H-X) \leqslant \Theta(H)-14=\Theta(H)-7|X|$, contradicting Claim II. Therefore, $d_{H^{\prime}}(y) \leqslant 1$ for every vertex $y \in N_{H}(x)$.

Since $\tau_{t}(H) \geqslant 3$ by Claim I, and since $H$ is connected, there exists a neighbor, $y^{*}$, of $x$ that has degree at least 1 in $H^{\prime}$. Let $X^{*}=\left\{x, y^{*}\right\}$ and consider the hypergraph $H^{*}=H-X^{*}$. Since $d_{H}(x)=3$ and $d_{H}\left(y^{*}\right) \geqslant 2$, the vertices $x$ and $y^{*}$ both contribute 2 to the sum $2 n(H)-n_{1}(H)$. Further since four distinct edges are deleted from $H$ when constructing $H^{*}$, the contribution of these deleted edges to the sum $2 m(H)$ is 8 .

Let $z \in N_{H}(x) \backslash\left\{y^{*}\right\}$. We note that if $z \in V\left(H^{*}\right)$, then $d_{H^{*}}(z) \leqslant d_{H^{\prime}}(z) \leqslant 1$, implying that $d_{H^{*}}(z)=1$ and that $z$ contributes 2 to the sum $2 n(H)-n_{1}(H)$ and 1 to the sum $2 n\left(H^{*}\right)-n_{1}\left(H^{*}\right)$. If $z \notin V\left(H^{*}\right)$, then $z$ is isolated when the vertices in $X^{*}$ are deleted from $H$ together with all edges incident with $X^{*}$, implying that in this case, $z$ contributes at least 1 to the sum $2 n(H)-n_{1}(H)$ and 0 to the sum $2 n\left(H^{*}\right)-n_{1}\left(H^{*}\right)$. In both cases the contribution of $z$ to $\Theta\left(H^{*}\right)$ is at least one less than its contribution to $\Theta(H)$. This is true for every vertex in $N_{H}(x) \backslash\left\{y^{*}\right\}$. Hence the total contribution of the neighbors of $x$ different from $y^{*}$ to $\Theta(H)-\Theta\left(H^{*}\right)$ is at least $\left|N_{H}(x) \backslash\left\{y^{*}\right\}\right|=\left|N_{H}(x)\right|-1 \geqslant k-1 \geqslant 4$. 
Together with our earlier observation that the vertices $x$ and $y^{*}$, together with the four edges incident with $x$ or $y^{*}$ in $H$, contribute 12 to $\Theta(H)$, this implies that $\Theta\left(H^{*}\right) \leqslant$ $\Theta(H)-12-4<\Theta(H)-14=\Theta(H)-7\left|X^{*}\right|$, contradicting Claim II. (口)

We now return to the proof of Theorem 28. By Claim IV, $\Delta(H)=2$. Let $x$ be a vertex in $H$ with $d_{H}(x)=2$. Since $\tau_{t}(H) \geqslant 3$ by Claim I, and since $H$ is connected, there exists an edge, $e$, that contains a neighbor, $y$, of $x$ but does not contain $x$. Let $X=\{x, y\}$ and consider the hypergraph $H-X$. Since $d_{H}(x)=2$ and $d_{H}(y)=2$, the vertices $x$ and $y$ both contribute 2 to the sum $2 n(H)-n_{1}(H)$. Further the three edges incident with $x$ or $y$ contribute 6 to the sum $2 m(H)$. Furthermore, each vertex in $N_{H}(x) \backslash\{y\}$ either does not belong to $H-X$ or has degree 1 in $H-X$ and therefore contributes at least 1 to $\Theta(H)-\Theta(H-X)$. This implies that $\Theta(H-X) \leqslant \Theta(H)-10-\left(\left|N_{H}(x)\right|-1\right) \leqslant$ $\Theta(H)-10-k+1 \leqslant \Theta(H)-14=\Theta(H)-7|X|$, contradicting Claim II. This completes the proof of Theorem 28 .

As an immediate consequence of Theorem 28, we have the following results.

Corollary 29. For $k \geqslant 5$, if $H \in \mathcal{H}_{k}$, then $7 \tau_{t}(H) \leqslant 2 n_{H}+2 m_{H}$.

Corollary 30. For all $k \geqslant 5$, we have $b_{k} \leqslant \frac{2}{7}$.

Theorem 4 follows from Corollary 24, Corollary 26 and Corollary 29.

\subsection{Proof of Theorem 6}

In this section, we present a proof of Theorem 6. Recall its statement.

Theorem 6. For $k \geqslant 4$, if $H \in \mathcal{H}_{k}^{*}$, then $\gamma_{t}(H) \leqslant\left(\max \left\{\frac{2}{k+2}, b_{k-1}\right\}\right) n_{H}$.

Proof of Theorem 6. Suppose to the contrary that the theorem is not true. Let $H \in \mathcal{H}_{k}^{*}$ be a counterexample with $n_{H}+m_{H}$ a minimum. We proceed in a similar manner as in the proof of Theorem 3 .

Claim I. The following properties hold in the hypergraph $H$.

(a) $H$ is connected.

(b) The deletion of any edge in $H$ creates an isolated vertex or an isolated edge.

(c) There is no dominating vertex in $H$.

Proof of Claim I. Parts (a) and (b) follows from the minimality of $H$ and the observation that the deletion of an edge cannot decrease the total domination number. To prove Part (c), suppose that $H$ contains a dominating vertex $v$. The vertex $v$ and any one of its neighbors forms a TD-set in $H$, implying that $\gamma_{t}(H)=2$. By the definition of $\mathcal{H}_{k}^{*}$, the hypergraph $H$ contains no isolated vertex or isolated edge. Since no two edges of $H$ intersect in $k-1$ vertices, we therefore have that $n_{H} \geqslant k+2$. Hence, $\gamma_{t}(H) \leqslant 2 n_{H} /(k+2)$, contradicting the definition of $H$. This proves Part (c). (口)

Claim II. Every edge in $H$ contains at least one degree-1 vertex. 
Proof of Claim II. We proceed as in the proof of Claim 20. Let $u, v, e, e_{1}$ and $e_{2}$ be defined as in the proof of Claim 20. If the edge $e_{2}$ contains a degree- 1 vertex, then at least one vertex in addition to the vertices in $e_{1} \cup\{u\}$ becomes an isolated vertex when we delete all edges incident with $u$ or $v$. Thus in this case we delete at least $k+2$ vertices and we add two vertices to $T$, and we proceed as in the 2nd paragraph of the proof of Claim 20. In this case, $|T|=2+\ell$, where $\ell \geqslant 0$ denotes the number of isolated edges created when removing $u$ and $v$, and at least $k+2+k \ell$ vertices are deleted from $H$. Thus if $n^{\prime}$ denotes the number of vertices in $H$ that are not deleted in the process, then $n^{\prime} \leqslant n_{H}-k-2-k \ell$, implying that

$$
\begin{aligned}
\left(\frac{2}{k+2}\right)\left(n_{H}-n^{\prime}\right) & \geqslant\left(\frac{2}{k+2}\right)(k+2+k \ell) \\
& =2+\left(\frac{2 k}{k+2}\right) \ell \\
& \geqslant 2+\ell \\
& =|T| .
\end{aligned}
$$

Suppose that the edge $e_{2}$ does not contain any degree- 1 vertices. Then there is an edge, $e_{3}$, which would become isolated after the deletion of the edge $e_{2}$ from $H_{2}$. We note that neither $u$ nor $v$ belong to the edge $e_{3}$ and therefore that $e_{3} \notin\left\{e, e_{1}, e_{2}\right\}$. Let $w \in e_{2} \cap e_{3}$. We now delete all edges incident with a vertex in the set $\{u, v, w\}$ and we delete any resulting isolated vertices. Further we add the three vertices $u, v$ and $w$ to the set $T$. We note that every vertex in $e_{1} \cup e_{3} \cup\{u\}$ becomes an isolated vertex. We therefore delete at least $2 k+1$ vertices and we add three vertices to $T$. If this process creates an isolated edge, then from each such isolated edge $f$, if any, we choose one vertex that is a neighbor of a vertex in $T$ and add it into $T$, and delete the $k$ vertices in $f$. Hence in this case, $|T|=3+\ell$, where $\ell \geqslant 0$ denotes the number of isolated edges created when removing $u, v$ and $w$, and at least $2 k+1+k \ell$ vertices are deleted from $H$. Thus if $n^{\prime}$ denotes the number of vertices in $H$ that are not deleted in the process, then $n^{\prime} \leqslant n_{H}-2 k-1-k \ell$. Since $k \geqslant 4$, we note that $2(2 k+1) /(k+2) \geqslant 3$ and $2 k /(k+2)>1$, implying that

$$
\begin{aligned}
\left(\frac{2}{k+2}\right)\left(n_{H}-n^{\prime}\right) & \geqslant\left(\frac{2}{k+2}\right)(2 k+1+k \ell) \\
& =\left(\frac{2(2 k+1)}{k+2}\right)+\left(\frac{2 k}{k+2}\right) \ell \\
& \geqslant 3+\ell \\
& =|T| .
\end{aligned}
$$

In both cases, we therefore have that $|T| \leqslant 2\left(n_{H}-n^{\prime}\right) /(k+2)$. If $n^{\prime}=0$, then the set $T$ is a TD-set in $H$, implying that $\gamma_{t}(H) \leqslant|T| \leqslant 2 n_{H} /(k+2)$, a contradiction. Hence, 
$n^{\prime}>0$. Let $H^{\prime}$ denote the resulting hypergraph on these $n^{\prime}$ vertices. Let $H^{\prime}$ have size $m^{\prime}$. By construction, the hypergraph $H^{\prime}$ is in the family $\mathcal{H}_{k}^{*}$. In particular, we note that $n^{\prime} \geqslant k+2$. By the minimality of $H$, we have that

$$
\gamma_{t}\left(H^{\prime}\right) \leqslant\left(\max \left\{\frac{2}{k+2}, b_{k-1}\right\}\right) n^{\prime}
$$

Let $T^{\prime}$ be a $\gamma_{t}\left(H^{\prime}\right)$-set and note that the set $T \cup T^{\prime}$ is a TD-set of $H$. Suppose that $2 /(k+2) \geqslant b_{k-1}$. Then, $\left|T^{\prime}\right| \leqslant 2 n^{\prime} /(k+2)$, and so

$$
\gamma_{t}(H) \leqslant\left|T \cup T^{\prime}\right| \leqslant\left(\frac{2}{k+2}\right)\left(n_{H}-n^{\prime}\right)+\left(\frac{2}{k+2}\right) n^{\prime}=\left(\frac{2}{k+2}\right) n_{H},
$$

a contradiction. Hence, $2 /(k+2)<b_{k-1}$. Thus, $\left|T^{\prime}\right| \leqslant b_{k-1} n^{\prime}$, and so

$$
\gamma_{t}(H) \leqslant\left|T \cup T^{\prime}\right| \leqslant\left(\frac{2}{k+2}\right)\left(n_{H}-n^{\prime}\right)+b_{k-1} n^{\prime}<b_{k-1}\left(n_{H}-n^{\prime}\right)+b_{k-1} n^{\prime}=b_{k-1} n_{H},
$$

a contradiction. This completes the proof of Claim II. (口)

We now return to the proof of Theorem 6. By Claim II, every edge in $H$ contains at least one degree-1 vertex. Let $H^{\prime}$ be the hypergraph obtained from $H$ by deleting exactly one degree- 1 vertex from each edge. Since $H \in \mathcal{H}_{k}^{*}$, we note that no multiple edges are created. Further, $H^{\prime}$ contains no isolated edge and no isolated vertices, and so $H^{\prime} \in \mathcal{H}_{k-1}$. Let $H^{\prime}$ have order $n^{\prime}$ and size $m^{\prime}$. Then, $n^{\prime}=n_{H}-m_{H}$ and $m^{\prime}=m_{H}$. Thus, $n^{\prime}+m^{\prime}=n_{H}$. We note that $k-1 \geqslant 3$. By Definition 2 we have that $\tau_{t}\left(H^{\prime}\right) \leqslant\left(n^{\prime}+m^{\prime}\right) b_{k-1} \leqslant b_{k-1} n_{H}$. An identical proof as in the proof of Claim 22 of Theorem 3 shows that $\gamma_{t}(H)=\tau_{t}\left(H^{\prime}\right)$, implying that $\gamma_{t}(H) \leqslant b_{k-1} n_{H}$, a contradiction. This completes the proof of Theorem 6 .

\section{Tight Asymptotic Bounds}

In this section we prove Theorem 8 which establishes a tight asymptotic upper bound on $b_{k}$ for $k$ sufficiently large. Since every strong transversal in a hypergraph, $H$, is a total transversal in $H$, and since every total transversal in $H$ is a transversal in $H$, we have the following observation.

Observation 31. For every hypergraph $H$, we have $\tau(H) \leqslant \tau_{t}(H) \leqslant \tau_{s}(H)$.

Using probabilistic arguments, Alon [3] established the following result.

Theorem 32. ([3]) For every $\varepsilon>0$ and sufficiently large $k$ there exist $k$-uniform hypergraphs, $H$, satisfying

$$
\tau(H) \geqslant\left(\frac{(1-\varepsilon) \ln (k)}{k}\right)\left(n_{H}+m_{H}\right)
$$

The following result establishes a tight asymptotic upper bound on the strong transversal number of a $k$-uniform hypergraph for $k$ sufficiently large. 
Theorem 33. For every constant $c>1$ and every $k$-uniform hypergraph $H$, we have

$$
\tau_{s}(H) \leqslant\left(\frac{\ln (k)+\ln (c)}{k-1}\right) n_{H}+\left(\frac{\ln (k)+\ln (c)}{c(k-1)}\right) m_{H}+\left(\frac{2}{c k}\right) m_{H} .
$$

Proof. Let $H=(V, E)$ and let $p=\ln (c k) /(k-1)$. Let $X_{1}$ be a random subset of $V(H)$ where a vertex $x$ is chosen to be in $X_{1}$ with probability $\operatorname{Pr}\left(x \in X_{1}\right)=p$, independently of the choice for any other vertex. For every edge $e \in E$ that does not intersect $X_{1}$, select two vertices from $e$ and let $X_{2} \subseteq V$ be the resulting set of all such selected vertices. For every edge $e \in E$ such that $\left|e \cap X_{1}\right|=1$, select one vertex from $e \backslash X_{1}$ and let $X_{3} \subseteq V$ be the resulting set of all such selected vertices. The resulting set $X_{1} \cup X_{2} \cup X_{3}$ is a strong transversal in $H$. The expected value of the cardinality of the set $X_{1}$ is

$$
\mathbb{E}\left(\left|X_{1}\right|\right)=p n_{H}=\left(\frac{\ln (k)+\ln (c)}{k-1}\right) n_{H} .
$$

Using the inequality $1-x \leqslant \mathrm{e}^{-x}$ for $x \in \mathbb{R}$, the expected value of the cardinality of the set $X_{2}$ is given by

$$
\begin{aligned}
\mathbb{E}\left(\left|X_{2}\right|\right) & \leqslant(1-p)^{k} \cdot m_{H} \cdot 2 \\
& =\left(1-\frac{\ln (c k)}{k-1}\right)^{k} \cdot 2 m_{H} \\
& =\left(\left(1-\frac{\ln (c k)}{k-1}\right)^{\frac{k-1}{\ln (c k)}}\right)^{\frac{k}{k-1} \ln (c k)} \cdot 2 m_{H} \\
& <\mathrm{e}^{-\frac{k}{k-1} \ln (c k)} \cdot 2 m_{H} \\
& \leqslant \frac{2}{c k} \cdot m_{H} .
\end{aligned}
$$

The expected value of the set $X_{3}$ is given by

$$
\begin{aligned}
\mathbb{E}\left(\left|X_{3}\right|\right) & \leqslant m_{H} \cdot k \cdot p \cdot(1-p)^{k-1} \\
& =k\left(\frac{\ln (c k)}{k-1}\right)\left(1-\frac{\ln (c k)}{k-1}\right)^{k-1} \cdot m_{H} \\
& =k\left(\frac{\ln (c k)}{k-1}\right)\left(\left(1-\frac{\ln (c k)}{k-1}\right)^{\frac{k-1}{\ln (c k)}}\right)^{\ln (c k)} \cdot m_{H} \\
& <k\left(\frac{\ln (c k)}{k-1}\right) \mathrm{e}^{-\ln (c k)} \cdot m_{H} \\
& =\left(\frac{\ln (k)+\ln (c)}{c(k-1)}\right) m_{H} .
\end{aligned}
$$


By linearity of expectation, we have that $\mathbb{E}\left(\left|X_{1} \cup X_{2} \cup X_{3}\right|\right) \leqslant \mathbb{E}\left(\left|X_{1}\right|\right)+\mathbb{E}\left(\left|X_{2}\right|\right)+$ $\mathbb{E}\left(\left|X_{3}\right|\right)$, yielding the desired upper bound.

As a consequence of Theorem 33, we have the following results.

Corollary 34. Given any $\varepsilon>0$, if $H$ is a $k$-uniform hypergraph with $k$ sufficiently large, then

$$
\tau_{s}(H)<\left((1+\varepsilon) \frac{\ln (k)}{k}\right)\left(n_{H}+m_{H}\right) .
$$

Proof. For a constant $c>1$, we note that the functions,

$$
\frac{\ln (k)+\ln (c)}{k-1} \text { and } \quad \frac{\ln (k)+\ln (c)}{c(k-1)}+\frac{2}{c k}
$$

tend to $\ln (k) /(k-1)$ and $\ln (k) /(c(k-1))<\ln (k) /(k-1)$, respectively, when $k$ tends to infinity. Hence for $k$ sufficiently large, we have that

$$
\max \left\{\frac{\ln (k)+\ln (c)}{k-1}, \frac{\ln (k)+\ln (c)}{c(k-1)}+\frac{2}{c k}\right\}<(1+\varepsilon) \frac{\ln (k)}{k} .
$$

Therefore for $k$ sufficiently large, we have that

$$
\left(\frac{\ln (k)+\ln (c)}{k-1}\right) n_{H}+\left(\frac{\ln (k)+\ln (c)}{c(k-1)}\right) m_{H}+\left(\frac{2}{c k}\right) m_{H}<\left((1+\varepsilon) \frac{\ln (k)}{k}\right)\left(n_{H}+m_{H}\right) .
$$

The desired result now follows from Theorem 33.

We are now in a position to prove Theorem 8. Recall its statement.

Theorem 8. $b_{k}=(1+\mathrm{o}(1)) \frac{\ln (k)}{k}$.

Proof of Theorem 8. It suffices for us to prove that for $\varepsilon>0$ and for $k$ sufficiently large, we have

$$
(1-\varepsilon) \frac{\ln (k)}{k} \leqslant b_{k} \leqslant(1+\varepsilon) \frac{\ln (k)}{k} .
$$

The upper bound on $b_{k}$ follows from Observation 31 and Corollary 34 . For the lower bound let $\varepsilon>0$ and let $k$ be sufficiently large, such that a $k$-uniform hypergraph, $H$, exists with $\tau(H) \geqslant[(1-\varepsilon) \ln (k) / k]\left(n_{H}+m_{H}\right)$ (which exists by Theorem 32). Assume that $H$ contains $n_{0}$ isolated vertices and $e_{0}$ isolated edges. Let $H^{\prime}$ be obtained from $H$ by deleting all isolated vertices and isolated edges and the vertices belonging to isolated edges. Then, $H^{\prime} \in \mathcal{H}_{k}$. Further, $n\left(H^{\prime}\right)=n_{H}-n_{0}-k e_{0}$ and $m\left(H^{\prime}\right)=m_{H}-e_{0}$. As $n_{0} \geqslant 0$ and $(1-\varepsilon) \ln (k)(k+1) / k>1$ when $k$ is sufficiently large, we have that 


$$
\begin{aligned}
\tau_{t}\left(H^{\prime}\right) & \geqslant \tau\left(H^{\prime}\right) \\
& =\tau(H)-e_{0} \\
& \geqslant\left(\frac{(1-\varepsilon) \ln (k)}{k}\right)\left(n_{H}+m_{H}\right)-e_{0} \\
& \geqslant\left(\frac{(1-\varepsilon) \ln (k)}{k}\right)\left(n\left(H^{\prime}\right)+m\left(H^{\prime}\right)+n_{0}+k e_{0}+e_{0}\right)-e_{0} \\
& =\left(\frac{(1-\varepsilon) \ln (k)}{k}\right)\left(n\left(H^{\prime}\right)+m\left(H^{\prime}\right)\right) \\
& \geqslant\left(\frac{(1-\varepsilon) \ln (k)}{k}\right)\left(n\left(H^{\prime}\right)+m\left(H^{\prime}\right)\right) .
\end{aligned}
$$

This implies that

$$
b_{k} \geqslant\left(\frac{(1-\varepsilon) \ln (k)}{k}\right),
$$

which establishes the desired lower bound on $b_{k}$ and completes the proof of Theorem 8 .

\section{Closing Remarks and Open Problem}

In view of Theorem 3 , it is of interest to determine the value of $b_{k}$ for $k \geqslant 2$. In Theorem 4 we show that $b_{2}=\frac{2}{5}$ and $b_{3}=\frac{1}{3}$, and we show that $b_{k-1} \leqslant 2 /(k+1)$ for $k \in\{3,4,5,6\}$. In Theorem 8, we establish a tight asymptotic bound on $b_{k}$ for $k$ sufficiently large which shows that is not true that $b_{k-1} \leqslant 2 /(k+1)$ when $k$ is large enough. We pose the following problems that still remain to be settled.

Problem 35. Determine the exact value of $b_{k}$ for $k \geqslant 4$.

Problem 36. Determine the smallest value of $k$ for which $b_{k-1}>2 /(k+1)$.

\section{Acknowledgements}

Research of Csilla Bujtás and Zsolt Tuza supported in part by the Hungarian Scientific Research Fund, OTKA grant T-81493, and by the European Union and Hungary, co-financed by the European Social Fund through the project TÁMOP-4.2.2.C-11/1/KONV-20120004 - National Research Center for Development and Market Introduction of Advanced Information and Communication Technologies. Research of Michael Henning supported in part by the South African National Research Foundation and the University of Johannesburg. 


\section{References}

[1] B. D. Acharya. Domination in hypergraphs. AKCE J. Combin., 4:117-126, 2007.

[2] B. D. Acharya. Domination in hypergraphs II. New directions. Proc. Int. Conf. ICDM, Mysore, India, pp. 1-16, 2008.

[3] N. Alon. Transversal numbers of uniform hypergraphs. Graphs Combin., 6:1-4, 1990.

[4] Cs. Bujtás, M. A. Henning and Zs. Tuza. Transversals and domination in uniform hypergraphs. European J. Combin., 33:62-71, 2012.

[5] V. Chvátal and C. McDiarmid. Small transversals in hypergraphs. Combinatorica, 12:19-26, 1992.

[6] E. J. Cockayne, R. M. Dawes, and S. T. Hedetniemi. Total domination in graphs. Networks, 10:211-219, 1980.

[7] M. Dorfling and M. A. Henning. Linear hypergraphs with large transversal number and maximum degree two. European J. Combin., 36:231-236, 2014.

[8] T. W. Haynes, S. T. Hedetniemi, and P. J. Slater (eds). Fundamentals of Domination in Graphs. Marcel Dekker, Inc. New York, 1998.

[9] T. W. Haynes, S. T. Hedetniemi, and P. J. Slater (eds). Domination in Graphs: Advanced Topics. Marcel Dekker, Inc. New York, 1998.

[10] M. A. Henning. Recent results on total domination in graphs: A survey. Discrete Math., 309:32-63, 2009.

[11] M. A. Henning and C. Löwenstein. Hypergraphs with large domination number and edge sizes at least 3. Discrete Applied Math., 160:1757-1765, 2012.

[12] M. A. Henning and C. Löwenstein. Hypergraphs with large transversal number and with edge sizes at least four. Central European J. Math., 10:1133-1140, 2012.

[13] M. A. Henning and A. Yeo. Hypergraphs with large transversal number and with edge sizes at least three. J. Graph Theory, 59:326-348, 2008.

[14] M. A. Henning and A. Yeo. Total domination in 2-connected graphs and in graphs with no induced 6-cycles. J. Graph Theory, 60:55-79, 2009.

[15] M. A. Henning and A. Yeo. Strong transversals in hypergraphs and double total domination in graphs. SIAM J. Discrete Math., 24:1336-1355, 2010.

[16] M. A. Henning and A. Yeo. Transversals and matchings in 3-uniform hypergraphs. European J. Combin., 34:217-228, 2013.

[17] M. A. Henning and A. Yeo. Total Domination in Graphs. Springer Monographs in Mathematics, ISBN-13: 978-1461465249 (2013).

[18] B. K. Jose, K. A. Germina, and K. Abhishek. On some open problems of stable sets and domination in hypergraphs. Preprint, 2009.

[19] B. K. Jose and Zs. Tuza. Hypergraph domination and strong independence. Applicable Analysis and Discrete Math., 3:237-358, 2009. 
[20] A. Kelmans and D. Mubayi. How many disjoint 2-edge paths must a cubic graph have? J. Graph Theory, 45:57-79, 2004.

[21] F. C. Lai and G. J. Chang. An upper bound for the transversal numbers of 4-uniform hypergraphs. J. Combin. Theory Ser. B, 50:129-133, 1990.

[22] S. Thomassé and A. Yeo. Total domination of graphs and small transversals of hypergraphs. Combinatorica, 27:473-487, 2007.

[23] Zs. Tuza. Covering all cliques of a graph. Discrete Math., 86:117-126, 1990. 